\title{
AN ANHARMONIC FOURIER ANALYSIS FOR USE IN PROBLEMS OF HEAT CONDUCTION AND MEIJER'S G-FUNCTION
}

\author{
S.D. BAJPAI AND SADHANA MISHRA
}

\begin{abstract}
In this paper, we develop an anharmonic Fourier analysis for use in problems of heat conduction. We also evaluate an integral involving Meijer's Gfunction and show how Meijer's G-function can be utilized to solve a problem of heat conduction in a rod under boundary conditions of special kind with the help of our anharmonic Fourier analysis.
\end{abstract}

\section{Introduction.}

The first object of this paper is to develop an anharmonic Fourier analysis for use in problems of heat conduction. The second object of the paper is to evaluate an integral involving Meijer's G-function and employ it to derive a solution of the equation of heat flow in a one-dimensional rod with constant thermal properties and no sources, under boundary conditions of special kind.

Cases in which heat is produced in the solid are becoming increasingly important in technical applications [13, pp. 11-12].

The use of special functions is becoming increasingly important in technical applications. Space research and nuclear reactor also given rise to several problems of the application of special functions. The application of the special functions associated with the names of Legendre, Bessel, Hermite, Laguerre, Jacobi etc. in applied problems were given by several mathematicians from time to time. Now, mathematicians, physicists and engineers strongly urge that the applications of the generalized hypergeometric functions should be attempted in the fields of applied mathematics, physical sciences and engineering. This paper is a humble contribution to satisfy that urge.

Most of the practical problems, where elementary functions are used, are capable of being generalized with help of generalized hypergeometric functions such as the generalized hypergeometric series, MacRobert's E-function, Meijer's G-function and Fox's H-function. These generalizations may appear only mathematically interesting, and not appear to give meaningful physical interpretations at present. Nonetheless, it is expected that extensive study of the generalized hypergeometric functions together with the development of computers in future will open new frontiers of their applications which are

Received March 28, 1989. 
hither to unknown. However, some particular cases of our results may lead to meaningful physical interpretations.

Bajpai [1-12], Kalla and Kushwaha [17], Prasad and Siddiqui [20,21], Prasad and Singh [22], Shah [23,24], Sharma [25], Singh and Varma [26] and some others [18, 19, 27] have used generalized hypergeometric functions in finding the solutions of one - dimensional boundary value problems relating heat conduction, electrostatic field between two spherical conductors, potential theory, forced symmetrical vibrations in a circular elastic plate, angular displacement in a shaft, free oscillations of water in a circular lake, transverse vibrations in a circular memberance etc.

Meijer's G-function is a generalization of almost all special functions appearing in physical sciences and engineering [15 p. 434-444]. Therefore, the solution obtained in this paper is of a general character and hence may encompass several cases of interest. Our solution is a master or key formula from which a large number of solutions may be obtained for MacRobert E-function, Hypergeometric functions, Bessel functions, Legendre functions, Whittaker functions, Orthogonal polynomials and other related higher transcendental functions [14, pp. 215-222]. The results so derived may be found of great value for computing different values of $f(x)$ for several special functions.

The following formulate are required in the proof.

The Meijer's G-function introduced and defined by Meijer will be represented as follow [14, p. 207, (1)] :

$$
\begin{aligned}
& G_{p, q}^{u, v}\left(\left.z\right|_{b_{1}, \ldots, b_{q}} ^{a_{1}, \ldots, a_{p}}\right) \\
= & \int_{C} \frac{\Pi_{j=1}^{u} \Gamma\left(b_{j}-s\right) \Pi_{j=1}^{v} \Gamma\left(1-a_{j}+s\right) z^{s}}{\Pi_{j=u+1}^{b} \Gamma\left(1-b_{j}+s\right) \Pi_{j=v+1}^{q} \Gamma\left(a_{j}-s\right)} d s,
\end{aligned}
$$

where $C$ is a suitable Barner contour.

The multiplication formula for the Gamma - function [14, p.4,(11)] :

$$
\Gamma(m z)=(2 \pi)^{\frac{1}{2}-\frac{1}{2} m} m^{m z-\frac{1}{2}} \prod_{i=0}^{m-1} \Gamma\left(z+\frac{i}{m}\right),
$$

where $m$ is a positive integer.

The following modified form of the integral [16, p.372,(8)]:

$$
\begin{aligned}
& \int_{0}^{L}\left(\sin \frac{\pi x}{L}\right)^{w-1} \cos \lambda_{m} \frac{\pi x}{L} d x \\
= & \frac{L \cos \lambda_{m} \frac{\pi}{2} \Gamma(w)}{2^{w-1} \Gamma\left(\frac{w+\lambda_{m}+1}{2}\right) \Gamma\left(\frac{w-\lambda_{m}+1}{2}\right)}, \quad \text { Rew }>0 .
\end{aligned}
$$

In what follows for sake of brevity $a_{p}$ stands for $a_{1}, \ldots, a_{p} ; d$ is a positive integer and the symbol $\Delta(d, w)$ represents the set of parameters $\frac{w}{d}, \frac{w+1}{d}, \cdots, \frac{w+d-1}{d}$. 


\section{Anharmonic Fourier analysis.}

We develop the following anharmonic Fourier analysis for use in problems of heat conduction. Generally, we consider half range Fourier series, which proceed according to harmonic (integral) overtones of a fundamental tone, e.g.

$$
f(x)=\frac{A_{0}}{2}+\sum_{n=1}^{\infty} A_{n} \cos n x \text { and } f(x)=\sum_{n=1}^{\infty} B_{n} \sin n x,
$$

We now consider the problem of expanding an arbitrary function $f(x)$ in the interval $0<x<\pi$ into a series of the form

$$
f(x)=\sum_{k=1}^{\infty} A_{k} \cos \lambda_{k} x
$$

where $\lambda_{k}$ is given as the roots of a transcendental equation, e.g.

$$
\cot \lambda \pi=\alpha \lambda, \quad \text { ( } \alpha \text { being an arbitrary number }) .
$$

The equation (3.2) has infinitely many roots.

We now show that the function $\cos \lambda_{k} x$ form an orthogonal system with weighting factor $g(x)=1$, i.e.

$$
\int_{0}^{\pi} \cos \lambda_{k} x \cos \lambda_{\ell} x d x= \begin{cases}0, & \text { if } k \neq \ell ; \\ \frac{\pi}{2}\left(1+\frac{\sin 2 \lambda_{k} \pi}{2 \lambda_{k} \pi}\right), & \text { if } k=\ell .\end{cases}
$$

When $k \neq \ell$, expressing from the product of cosines to the cosines of surns and differences, we obtain from the left hand side of (3.3) the given expression:

$$
\frac{\lambda_{k} \lambda_{\ell}}{\lambda_{k}^{2}-\lambda_{\ell}^{2}} \sin \lambda_{k} \pi \sin \lambda_{\ell} \pi\left[\frac{\cot \lambda_{\ell} \pi}{\lambda_{\ell}}-\frac{\cot \lambda_{k} \pi}{\lambda_{k}}\right],
$$

where the expression inside the brackets vanishes because of (3.2).

When $k=\ell$, similarly from (3.3), we obtain

$$
\int_{0}^{\pi} \cos ^{2} \lambda_{k} x d x=\frac{\pi}{2}\left(1+\frac{\sin 2 \lambda_{k} \pi}{2 \lambda_{k} \pi}\right) .
$$

From (3.1) and (3.3), we easily find the following value for the Fourier coefficients $A_{k}$ as:

$$
A_{k}=\frac{2}{\pi} \int_{0}^{\pi} \frac{f(x) \cos \lambda_{k} x}{1+\frac{\sin 2 \lambda_{k} \pi}{2 \lambda_{k} \pi}} d x
$$

2. The integral. 
The integral to be evaluated is

$$
\begin{aligned}
& \int_{0}^{L}\left(\sin \frac{\pi x}{L}\right)^{w-1} \cos \lambda_{m} \frac{\pi x}{L} G_{p, q}^{u, v}\left(\left.z\left(\sin \frac{\pi x}{L}\right)^{2 d}\right|_{b_{q}} ^{a_{p}}\right) d x \\
& =\frac{L \cos \frac{\pi}{2} \lambda_{m}}{\sqrt{(\pi d)}} G_{p+2 d, q+2 d}^{u, v+2 d}\left(\left.z\right|_{b_{q}, \Delta\left(d, \frac{1-w-\lambda_{m}}{2}\right), \Delta\left(d, \frac{1-w+\lambda_{m}}{2}\right)} ^{\Delta(2 d, 1-w), a_{p}}\right) \\
& \text { where } 2(u+v)>p+q,|\arg z|<\left(u+v-\frac{1}{2} p-\frac{1}{2} q\right) \pi \\
& \operatorname{Re}\left(w+2 d b_{j}\right)>0, \quad(j=1, \cdots, u) .
\end{aligned}
$$

Proof. To establish the integral, expressing the G-function in the integrand as a Mellin-Barnes type integral (1.1) and interchanging the order of intergrations, which is justified due to absolute convergence of the integrals involved in the process, we get

$$
\frac{1}{2 \pi i} \int_{C} \frac{\Pi_{j=1}^{u} \Gamma\left(b_{j}-s\right) \Pi_{j=1}^{v} \Gamma\left(1-a_{j}+s\right) z^{s}}{\Pi_{j=u+1}^{q} \Gamma\left(1-b_{j}+s\right) \Pi_{j=v+1}^{p} \Gamma\left(a_{j}-s\right)} \int_{0}^{L}\left(\sin \frac{\pi x}{L}\right)^{w+2 s d-1} \cos \lambda_{m} \frac{\pi x}{L} d x d s .
$$

Now evaluating the inner-integral with the help of (1.3), and using the multiplication formula for the gamma-function, we have

$$
\begin{aligned}
& \frac{L \cos \frac{\pi}{2} \lambda_{m}}{\sqrt{(\pi d)}} \times \int_{C}\left\{\Pi_{j=1}^{u} \Gamma\left(b_{j}-s\right) \Pi_{j=1}^{v} \Gamma\left(1-a_{j}+s\right) \Pi_{i=0}^{2 d-1} \Gamma\left(\frac{w+i}{2 d}+s\right) z^{s}\right\} / \\
& \left\{\Pi_{j=u+1}^{q} \Gamma\left(1-b_{j}+s\right) \Pi_{j=v+1}^{p} \Gamma\left(a_{j}-s\right) \Pi_{i=0}^{d-1} \Gamma\left(\frac{\frac{\left(w+\lambda_{m}+1\right)}{2}+i}{d}+s\right)\right. \\
& \left.\Pi_{i=0}^{d-1} \Gamma\left(\frac{\frac{\left(w-\lambda_{m}+1\right)}{2}+i}{d}+s\right)\right\} d s .
\end{aligned}
$$

On applying (1.1), the value of the integral (2.1) is obtained. 


\section{Heat condition in a rod.}

Let us now consider a simple but typical problem of the so-called outer heat conduction in a rod. If the thermal coefficients are constant and there are no sources of thermal energy, then the temperature in a one-dimensional rod $0 \leq x \leq L$ satisfies :

$$
\frac{\partial u}{\partial t}=K \frac{\partial^{2} u}{\partial x^{2}}, \quad t \geq 0
$$

The solution of this partial differential equation must satisfy the following condition

$$
\begin{gathered}
\frac{\partial u}{\partial x}(0, t)=0 \\
u(L, t)+h \frac{\partial u}{\partial x}(L, t)=0 \\
u(x, t) \text { is finite when } t \rightarrow \infty . \\
u(x, 0)=f(x)=\left(\sin \frac{\pi x}{L}\right)^{w-1} G_{p, q}^{u, v}\left(\left.z\left(\sin \frac{\pi x}{L}\right)^{2 d}\right|_{b_{q}} ^{a_{p}}\right) .
\end{gathered}
$$

\section{Solution of the problem.}

The solution of the problem to be obtained is

$$
\begin{aligned}
& u(x, t)=\frac{2}{\sqrt{(\pi d)}} \sum_{n=1}^{\infty} \frac{\cos \frac{\pi}{2} \lambda_{n} \cos \lambda_{n} \frac{\pi x}{L} e^{-\left(\pi \lambda_{n} / L\right)^{2} k t}}{\left(1+\frac{1}{2 \pi \lambda_{n}} \sin 2 \pi \lambda_{n}\right)} \\
& \times G_{p+2 d, q+2 d}^{u, v+2 d}\left(\left.z\right|_{b_{q}, \Delta\left(d, \frac{1-w-\lambda_{n}}{2}\right), \Delta\left(d, \frac{1-w+\lambda_{n}}{2}\right)} ^{\Delta(2 d, 1-w), a_{p}}\right), \\
& \text { where } 2(u+v)>p+q,|\arg z|<\left(u+v-\frac{1}{2} p-\frac{1}{2} q\right) \pi, \\
& \operatorname{Re}\left(w+2 d b_{j}\right)>0, \quad(j=1, \cdots, u) .
\end{aligned}
$$

Proof. We now wish to treat the boundary conditions (4.2) and (4.3). In order to satisfy (4.2), we set

$$
f(x)=\sum_{n=1}^{\infty} A_{n} \cos \lambda_{n} \pi \frac{x}{L}
$$


That is to say in the solution of $\frac{\partial u}{\partial x}(0, t)=0$ and $\frac{\partial u}{\partial x}(L, t)=0$, we replace the sequence of integers $n$ by the sequence:

$$
\lambda_{1}, \lambda_{2}, \ldots, \lambda_{n}, \ldots,
$$

which we wish to determine in such a way that the condition (4.3) is satisfied. This leads to the transcendental equation

or

$$
\cos \lambda_{n} \pi-\lambda_{n} \frac{h \pi}{L} \sin \lambda_{n} \pi=0
$$

$$
\cot \lambda_{n} \pi=\lambda_{n} \frac{h \pi}{L} .
$$

This is equation (3.2) with $\alpha=\frac{h \pi}{L}$. Hence we are dealing here with a typical case of anharmonic Fourier analysis discussed in section 3. We obtain the final solution of our boundary value problem (4.2) and (4.3), if we multiply the terms in the sums of (5.2) by the required time factor, viz. $\exp \left\{-\left(\frac{\lambda_{n} \pi}{L}\right)^{2} k t\right\}$. Hence our solution is

$$
u(x, t)=\sum_{n=1}^{\infty} A_{n} \cos \lambda_{n} \pi \frac{x}{L} e^{-\left(\pi \lambda_{n} / L\right)^{2} k t} .
$$

If $t=0$, then by virture of (4.5) and (5.4) we have

$$
\left(\sin \frac{\pi x}{L}\right)^{w-1} G_{p, q}^{u, v}\left(\left.z\left(\sin \frac{\pi x}{L}\right)^{2 d}\right|_{b_{q}} ^{a_{p}}\right)=\sum_{n=1}^{\infty} A_{n} \cos \lambda_{n} \frac{\pi x}{L} .
$$

Multiplying both sides of (5.5) by $\cos \lambda_{m} \frac{\pi x}{L}$ and integrating with respect to $x$ from 0 to $L$, we get

$$
\begin{aligned}
& \int_{0}^{L}\left(\sin \frac{\pi x}{L}\right)^{w-1} \cos \lambda_{m} \frac{\pi x}{L} G_{p, q}^{u, v}\left(\left.z\left(\sin \frac{\pi x}{L}\right)^{2 d}\right|_{b_{q}} ^{a_{p}}\right) d x \\
& =\sum_{n=1}^{\infty} A_{n} \int_{0}^{L} \cos \lambda_{n} \frac{\pi x}{L} \cos \lambda_{m} \frac{\pi x}{L} d x .
\end{aligned}
$$

Now using (2.1) and (3.3), we have

$$
\begin{aligned}
A_{m} & =\frac{2 \cos \lambda_{m} \frac{\pi}{2}}{\sqrt{(\pi d)}\left(1+\frac{1}{2 \lambda_{m} \pi} \sin 2 \lambda_{m} \pi\right)} \\
& \times G_{p+2 d, q+2 d}^{u, v+2 d}\left(\left.z\right|_{b_{q}, \Delta\left(d, \frac{1-w-\lambda_{m}}{\Delta(2 d, 1-w), a_{p}}\right.} ^{2}\right), \Delta\left(d, \frac{1-w+\lambda_{m}}{2}\right)
\end{aligned}
$$

With the help of (5.4) and (5.6), the solution (5.1) is obtained. 


\section{References}

[1] Bajpai, S.D. Use of Gauss's hypergeometric functions and Meijer's G-function is the production of heat in a cylinder. Proc. Camb. Phil. Soc. 64, (1968), 1049-1054.

[2] Bajpai, S.D. The use of Meijer's G-function in the electrostatic field between two spherical conductors. J. Sci. Engg. Res. XIII-1, (1969), 149-152.

[3] Bajpai, S.D. Meijer's G-function and temperature in a slab with faces at temperature zero. J. Sci. Engg. Res. XIII-2, (1969), 254-257.

[4] Bajpai, S.D. The potential about a spherical surface and Meijer's G-function, Vijnana Parishad Anusandhan Patrika, 12(11), (1969), 93-97.

[5] Bajpai, S.D. Meijer's G-function and the temperature in a non-homogeneous bar. Proc. Indian Acad. Sci. LXX, (1969), 697-701.

[6] Bajpai, S.D. Special functions and forced symmetrical vibrations in a circular elastic plate. Proc. Comb. Phi. Soc. 66, (1969), 349-353.

[7] Bajpai, S.D. An integral involving Fox's H-function and heat conduction. Math. Education Sect A, 7, (1969), 1-4.

[8] Bajpai, S.D. Fox's H-fuction and temperature in a slab with insulated faces. Jour. Math. Phys. Sci. 4, (1970), 302-307.

[9] Bajpai, S.D. Meijer's G-function and angular displacement in a shaft. Proc. Nat. Acad. Sci. India, 4.1 (A), (1971), 320-231.

[10] Ba.jpai, S.D. Meijer's G-function and temperature in a slab with thermally insulated faces. Proc. Int. Con. Num. Method. in Thermal problems II, (1981), 677-683.

[11] Bajpai, S.D. Fox's H-function and temperature in a non-homogenious bar. Proc. Int. Con. Num. Method in Thermal Problems III, (1983), 239-341.

[12] Bajpai, S.D. Fox's H-function and angular twist in a shaft. Proc. SAMSA Symposium V, (1986), 122-131.

[13] Carslaw, H.S. and Jaeger, J.C. Conduction of heat in solids. Clarendon Press, Oxford, (1986).

[14] Erdélyi, A. et al. Higher transcendental functions, Vol.1. McGraw Hill, New York, (1953).

[15] Erdélyi, A. et al. Tables of Integral transforms, Vol.2. McGraw Hill, (1954).

[16] Gradshteyn, I.S. and Ryzhik, I.M. Tables of Integrals, series and products. Academic Press, Inc., New York, (1980).

[17] Kalla, S.K. and Kushwaha, R.S. Production of heat in a cylinder. Acta Mexicana Cien. Tecn. 4, (1970), 89-93.

[18] Mathai, A.M. and Saxena, R.K. Lecture Notes in Maths. 348-Generalized hypergeometric functions with applications in statistics and physical sciences. Springer-Verlag Berlin, Heidelberg, (1973).

[19] Mathai, A.M. and Saxena, R.K. The H-function with applications in statistics and other disciplines. Wiley Eastern Ltd, New Delhi, (1978).

[20] Prasad, Y.N. and Siddiqui, A. Application of generalized function in the production of heat in a cylinder. Defence Sci. J. 25, (1975), 107-114.

[21] Prasad, Y.N. and Siddiqui, A. Application of generalized functions of two variables in vibrations of a circular memberance. Vijnana Parishad Anusandhan Patrika 20, (1977), 113-122.

[22] Prasad, Y.N. and Singh, S.N. An application of the H-function of several complex variables in the production of heat in a cylinder. Pure Appl. Math. Sci. 6, (1977), 57-64.

[23] Shah, M. Heat conduction, generalized Meijer function and Hermite polynomials. Comment Math. Univ. St. Paul. 18, (1970), 81-94.

[24] Shah, M. On generalized Meijer function of two variables and some applications. Comment. Math. Univ. St. Paul 19, (1970), 93-122.

[25] Sharma, O.P. H-function and heat production in a cylinder. Proc. Nat. Acad. Sci. India, Sect. A, 39, (1969), 355-360. 
[26] Singh, F. and Varma, R.C. Application of E-operator to evaluate a definite integral and its application in heat condition. J. Indian Math. Soc. 36, (1972), 325-332.

[27] Srivastava, H.M. , Gupta, K.C. and Goyal, S.P. The H-function of one and two variables with applications. South Asian Publishers, New Delhi, (1982).

S.D. Bajpai

Department of mathematics, University of Bahrain, P.O. Box 32038, Isa Town, BAHRAIN.

\section{Sadhana Mishra}

11, Sardarpura, Udaipur, INDIA. 